Теорія Ймовір. та Матем. Статист. Вип. 75, 2006
Theor. Probability and Math. Statist. No. 75, 2007, Pages 93-108 S 0094-9000(08)00717-5

Article electronically published on January 24, 2008

\title{
STOCHASTIC INTEGRALS AND STOCHASTIC DIFFERENTIAL EQUATIONS WITH RESPECT TO THE FRACTIONAL BROWNIAN FIELD
}

UDC 519.21

\author{
YU. S. MISHURA AND S. A. IL'CHENKO
}

\begin{abstract}
Stochastic differential equations on the plane are considered with respect to the fractional Brownian field. We prove the existence and uniqueness of a solution for such equations. These results are based on new estimates obtained for norms in the Besov type spaces for the two-parameter stochastic integral considered with respect to the fractional Brownian field.
\end{abstract}

\section{INTRODUCTION}

In this section, we consider Besov type spaces for two-parameter random fields on the plane and study generalized Lebesgue-Stieltjes integrals with integrands and integrators belonging to some Besov type space.

Let $T=\left(T_{1}, T_{2}\right) \in \mathbb{R}_{+}^{2}$ be a fixed point and let $f:[0, T]:=\left[0, T_{1}\right] \times\left[0, T_{2}\right] \rightarrow \mathbb{R}$ be a measurable function. We define the forward and backward fractional derivatives of the function $f$ of orders $\alpha_{i} \in(0,1), i=1,2$ :

$$
\begin{aligned}
D_{a+}^{\alpha_{1} \alpha_{2}} f(t):= & \frac{1}{\Gamma\left(1-\alpha_{1}\right) \Gamma\left(1-\alpha_{2}\right)} \\
& \times\left(\frac{f(t)}{\left(t_{1}-a_{1}\right)^{\alpha_{1}}\left(t_{2}-a_{2}\right)^{\alpha_{2}}}+\frac{\alpha_{1}}{\left(t_{2}-a_{2}\right)^{\alpha_{2}}} \int_{a_{1}}^{t_{1}} \frac{f(t)-f\left(s_{1}, t_{2}\right)}{\left(t_{1}-s_{1}\right)^{1+\alpha_{1}}} d s_{1}\right. \\
& \quad+\frac{\alpha_{2}}{\left(t_{1}-a_{1}\right)^{\alpha_{1}}} \int_{a_{2}}^{t_{2}} \frac{f(t)-f\left(t_{1}, s_{2}\right)}{\left(t_{2}-s_{2}\right)^{1+\alpha_{2}}} d s_{2} \\
& \left.\quad+\alpha_{1} \alpha_{2} \int_{[a, t]} \frac{\Delta_{s} f(t)}{\left(t_{1}-s_{1}\right)^{1+\alpha_{1}}\left(t_{2}-s_{2}\right)^{1+\alpha_{2}}} d s\right) \cdot \mathbf{1}_{[a, T]}(t),
\end{aligned}
$$

where $a \in[0, T],[a, t]=\left[a_{1}, t_{1}\right] \times\left[a_{2}, t_{2}\right], \Delta_{s} f(t)=f(t)-f\left(t_{1}, s_{2}\right)-f\left(s_{1}, t_{2}\right)+f(s)$, and

$$
\begin{aligned}
D_{b-}^{\alpha_{1} \alpha_{2}} f(t):= & \frac{1}{\Gamma\left(1-\alpha_{1}\right) \Gamma\left(1-\alpha_{2}\right)} \\
& \times\left(\frac{f(t)}{\left(b_{1}-t_{1}\right)^{\alpha_{1}}\left(b_{2}-t_{2}\right)^{\alpha_{2}}}+\frac{\alpha_{1}}{\left(b_{2}-t_{2}\right)^{\alpha_{2}}} \int_{t_{1}}^{b_{1}} \frac{f(t)-f\left(s_{1}, t_{2}\right)}{\left(s_{1}-t_{1}\right)^{1+\alpha_{1}}} d s_{1}\right. \\
& +\frac{\alpha_{2}}{\left(b_{1}-t_{1}\right)^{\alpha_{1}}} \int_{t_{2}}^{b_{2}} \frac{f(t)-f\left(t_{1}, s_{2}\right)}{\left(s_{2}-t_{2}\right)^{1+\alpha_{2}}} d s_{2} \\
& \left.\quad+\alpha_{1} \alpha_{2} \int_{[t, b]} \frac{\Delta_{s} f(t)}{\left(s_{1}-t_{1}\right)^{1+\alpha_{1}}\left(s_{2}-t_{2}\right)^{1+\alpha_{2}}} d s\right) \cdot \mathbf{1}_{[0, b]}(t),
\end{aligned}
$$

assuming that all the above integrals exist.

2000 Mathematics Subject Classification. Primary 60H10, 60H05, 60G15.

The first author is supported by the grant NATO PST.CLG 980408. 
Let $g_{t-}(s):=\Delta_{s} g(t), 0 \leq s \leq t \leq T$. The generalized fractional two-parameter Lebesgue-Stieltjes integral for the functions $f, g:[0, T] \rightarrow \mathbb{R}$ is defined by

$$
\int_{[0, T]} f d g=\int_{[0, T]} D_{0+}^{\alpha_{1} \alpha_{2}} f(t) D_{T-}^{1-\alpha_{1} 1-\alpha_{2}} g_{T-}(t) d t
$$

provided the right hand side is well defined as the Lebesgue integral. Properties of this integral are studied in the paper [1].

Now we introduce the following classes of functions.

Definition 1. A function $f:[0, T] \rightarrow \mathbb{R}$ belongs to the class $\mathcal{H}^{\lambda_{1}, \lambda_{2}}$ with $0<\lambda_{i} \leq 1$, $i=1,2$ (in other words, $f$ is a Hölder function of orders $\lambda_{1}$ and $\lambda_{2}$ on $[0, T]$ ) if there exists a constant $C>0$ such that

$$
\begin{gathered}
\left|\Delta_{s} f(t)\right| \leq C \prod_{i=1,2}\left|t_{i}-s_{i}\right|^{\lambda_{i}}, \\
\left|f(t)-f\left(s_{1}, t_{2}\right)\right| \leq C\left|t_{1}-s_{1}\right|^{\lambda_{1}}, \\
\left|f(t)-f\left(t_{1}, s_{2}\right)\right| \leq C\left|t_{2}-s_{2}\right|^{\lambda_{2}}
\end{gathered}
$$

for all $s \leq t, s, t \in[0, T]$.

Put

$$
\begin{aligned}
& r(f)(t):=|f(t)|+\int_{0}^{t_{1}} \frac{\left|f(t)-f\left(s_{1}, t_{2}\right)\right|}{\left(t_{1}-s_{1}\right)^{1+\alpha_{1}}} d s_{1}+\int_{0}^{t_{2}} \frac{\left|f(t)-f\left(t_{1}, s_{2}\right)\right|}{\left(t_{2}-s_{2}\right)^{1+\alpha_{2}}} d s_{2} \\
& +\int_{[0, t]} \frac{\left|\Delta_{s} f(t)\right|}{\left(t_{1}-s_{1}\right)^{1+\alpha_{1}}\left(t_{2}-s_{2}\right)^{1+\alpha_{2}}} d s, \\
& \|f\|:=\sup _{s \in[0, T]}|f(s)| \\
& \|f\|_{\lambda_{1}, \lambda_{2}}:=\|f\| \\
& +\sup _{0 \leq s \leq t \leq T}\left(\frac{\left|f\left(t_{1}, s_{2}\right)-f(s)\right|}{\left(t_{1}-s_{1}\right)^{\lambda_{1}}}+\frac{\left|f\left(s_{1}, t_{2}\right)-f(s)\right|}{\left(t_{2}-s_{2}\right)^{\lambda_{2}}}+\frac{\left|\Delta_{s} f(t)\right|}{\left(t_{1}-s_{1}\right)^{\lambda_{1}}\left(t_{2}-s_{2}\right)^{\lambda_{2}}}\right) .
\end{aligned}
$$

Following the paper [2] we introduce the following definitions.

Definition 2. A function $f:[0, T] \rightarrow \mathbb{R}$ belongs to the class

(1) $W_{1}^{\alpha_{1}, \alpha_{2}}$ if

$$
\|f\|_{1, \alpha_{1}, \alpha_{2}}:=\sup _{0 \leq t \leq T} r(f)(t)<\infty
$$

(2) $W_{2}^{\beta_{1}, \beta_{2}}$ if

$$
\begin{aligned}
\|g\|_{2, \beta_{1}, \beta_{2}}:=\sup _{0 \leq s<t \leq T}( & \frac{\left|\Delta_{s} g(t)\right|}{\left(t_{1}-s_{1}\right)^{\beta_{1}}\left(t_{2}-s_{2}\right)^{\beta_{2}}} \\
& +\frac{1}{\left(t_{2}-s_{2}\right)^{\beta_{2}}} \int_{s_{1}}^{t_{1}} \frac{\left|g_{t-}\left(u, s_{2}\right)-g_{t-}(s)\right|}{\left(u-s_{1}\right)^{1+\beta_{1}}} d u \\
& \quad+\frac{1}{\left(t_{1}-s_{1}\right)^{\beta_{1}}} \int_{s_{2}}^{t_{2}} \frac{\left|g_{t-}\left(s_{1}, v\right)-g_{t-}(s)\right|}{\left(v-s_{2}\right)^{1+\beta_{2}}} d v \\
& \left.\quad+\int_{[s, t]} \frac{\left|\Delta_{s} g(u, v)\right|}{\left(u-s_{1}\right)^{1+\beta_{1}}\left(v-s_{2}\right)^{1+\beta_{2}}} d u d v\right) \\
<\infty ; &
\end{aligned}
$$


(3) $W_{3}^{\gamma_{1}, \gamma_{2}}$ if

$$
\begin{aligned}
\|f\|_{3, \gamma_{1}, \gamma_{2}}:=\sup _{0 \leq s \leq t \leq T}\left[\int_{s_{1}}^{t_{1}} \int_{s_{2}}^{t_{2}}\right. & \left(\frac{|f(r)|}{\left(r_{1}-s_{1}\right)^{\gamma_{1}}\left(r_{2}-s_{2}\right)^{\gamma_{2}}}\right. \\
& +\frac{1}{\left(r_{2}-s_{2}\right)^{\gamma_{2}}} \int_{s_{1}}^{r_{1}} \frac{\left|f(r)-f\left(u, r_{2}\right)\right|}{\left(r_{1}-u\right)^{\gamma_{1}+1}} d u \\
& +\frac{1}{\left(r_{1}-s_{1}\right)^{\gamma_{1}}} \int_{s_{2}}^{r_{2}} \frac{\left|f(r)-f\left(r_{1}, v\right)\right|}{\left(r_{2}-v\right)^{\gamma_{2}+1}} d v \\
& \left.\left.+\int_{s_{1}}^{r_{1}} \int_{s_{2}}^{r_{2}} \frac{\left|\Delta_{u, v} f(r)\right|}{\left(r_{1}-u\right)^{\gamma_{1}+1}\left(r_{2}-v\right)^{\gamma_{2}+1}} d u d v\right) d r\right]<\infty
\end{aligned}
$$

Remark 1. The norm

$$
\|f\|_{\alpha_{1}, \alpha_{2}, \lambda_{1}, \lambda_{2}}:=\sup _{0 \leq t \leq T} e^{-\lambda_{1} t_{1}-\lambda_{2} t_{2}} r(f)(t), \quad \lambda_{i} \geq 0,
$$

is equivalent to the norm $\|\cdot\|_{1, \alpha_{1}, \alpha_{2}}$ on the space $W_{1}^{\alpha_{1}, \alpha_{2}}$.

Remark 2. It is obvious that

$$
\mathcal{H}^{\alpha_{1}+\varepsilon_{1}, \alpha_{2}+\varepsilon_{2}} \subset W_{1}^{\alpha_{1}, \alpha_{2}} \subset \mathcal{H}^{\alpha_{1}-\varepsilon_{1}, \alpha_{2}-\varepsilon_{2}}
$$

for all $0<\varepsilon_{i}<\alpha_{i}$ and that

$$
\mathcal{H}^{\beta_{1}+\varepsilon_{1}, \beta_{2}+\varepsilon_{2}} \subset W_{2}^{\beta_{1}, \beta_{2}} \subset \mathcal{H}^{\beta_{1}, \beta_{2}}
$$

for all $\varepsilon_{i}>0$.

Remark 3. It is worth mentioning that $\|g\|_{2, \beta_{1}, \beta_{2}}$ is not a norm in a usual sense.

Remark 4. For all $t \leq T$,

$$
\begin{aligned}
\left|\int_{[0, t]} f d g\right| & \leq \sup _{0<s<t}\left|\left(D_{t-}^{1-\alpha_{1} 1-\alpha_{2}} g_{t-}\right)(s)\right| \int_{[0, t]}\left|\left(D_{0+}^{\alpha_{1} \alpha_{2}} f\right)(s)\right| d s \\
& \leq \Lambda_{\alpha_{1}, \alpha_{2}}(g)\|f\|_{3, \alpha_{1}, \alpha_{2}} \leq C\|g\|_{2,1-\alpha_{1}, 1-\alpha_{2}}\|f\|_{3, \alpha_{1}, \alpha_{2}}
\end{aligned}
$$

where

$$
\Lambda_{\alpha_{1}, \alpha_{2}}(g):=\sup _{0<s \leq t}\left|\left(D_{t-}^{1-\alpha_{1} 1-\alpha_{2}} g_{t-}\right)(s)\right| .
$$

The constant $C$ depends on $\alpha_{1}$ and $\alpha_{2}$ only.

The text is organized as follows. Section 2 contains the proof of estimates for the norms in Besov type spaces for generalized two-parameter stochastic integrals with respect to the fractional Brownian field on the plane; namely, we estimate the norm of an integral in terms of the norm of its integrand. We apply this estimate to the case where the integrand is a functional of the fractional Brownian field on the plane, while the integrator is the fractional Brownian field itself. We further use these estimates for the construction of a solution of a stochastic differential equation with respect to the fractional Brownian field.

Section $[3$ is devoted to the proof of the existence and uniqueness of a solution of a stochastic differential equation with respect to the fractional Brownian field. 


\section{Estimates for Generalized TWO-PARAMETER STOCHAStiC LEBESGUE AND LEBESGUE-STIELTJES INTEGRALS}

Consider the function spaces of the Besov type introduced in the preceding section. We study the integral

$$
G_{t}(f):=\int_{[0, t]} f d g, \quad t \in[0, T] .
$$

The following result is proved in 3 .

Theorem $1(3])$.

(1) Let $g \in W_{2}^{1-\alpha_{1}, 1-\alpha_{2}}$ and $f \in W_{3}^{\alpha_{1}, \alpha_{2}}$. Then

$$
\left|\Delta_{s} G_{t}(f)\right| \leq \Lambda_{\alpha_{1}, \alpha_{2}}(g)\|f\|_{3, \alpha_{1}, \alpha_{2}}
$$

and

$$
\begin{aligned}
\left|G_{t}(f)\right| & +\int_{0}^{t_{1}} \frac{\left|G_{t}(f)-G_{s_{1}, t_{2}}(f)\right|}{\left(t_{1}-s_{1}\right)^{\alpha_{1}+1}} d s_{1}+\int_{0}^{t_{2}} \frac{\left|G_{t}(f)-G_{t_{1}, s_{2}}(f)\right|}{\left(t_{2}-s_{2}\right)^{\alpha_{2}+1}} d s_{2} \\
& +\int_{0}^{t_{1}} \int_{0}^{t_{2}} \frac{\left|\Delta_{s} G_{t}(f)\right|}{\left(t_{1}-s_{1}\right)^{\alpha_{1}+1}\left(t_{2}-s_{2}\right)^{\alpha_{2}+1}} d s \\
\leq & C_{\alpha_{1}, \alpha_{2}, T} \Lambda_{\alpha_{1}, \alpha_{2}}(g) \\
& \times \int_{0}^{t_{1}} \int_{0}^{t_{2}}\left(z_{1}^{-\alpha_{1}}+\left(t_{1}-z_{1}\right)^{-2 \alpha_{1}}\right)\left(z_{2}^{-\alpha_{2}}+\left(t_{2}-z_{2}\right)^{-2 \alpha_{2}}\right) r(f)(z) d z
\end{aligned}
$$

for all $0 \leq s<t \leq T$.

(2) If $g \in W_{2}^{\overline{1-}-\alpha_{1}, 1-\overline{\alpha_{2}}}$ and $f \in W_{1}^{\alpha_{1}, \alpha_{2}}$, then $G$. $(f) \in \mathcal{H}^{1-\alpha_{1}, 1-\alpha_{2}}$ and

$$
\|G(f)\|_{1-\alpha_{1}, 1-\alpha_{2}} \leq C_{\alpha_{1}, \alpha_{2}, T}^{*} \Lambda_{\alpha_{1}, \alpha_{2}}(g)\|f\|_{1, \alpha_{1}, \alpha_{2}} .
$$

The constants $C_{\alpha_{1}, \alpha_{2}, T}$ and $C_{\alpha_{1}, \alpha_{2}, T}^{*}$ in relations (3) and (41) depend on $\alpha_{1}, \alpha_{2}$, and T only.

Consider the superposition $\sigma(s, f(s))$ of a smooth function $\sigma$ and a function $f$ belonging to some Besov type space. The following result establishes the existence of the integral

$$
\int_{[0, t]} \sigma(s, f(s)) d g(s) .
$$

Theorem $2([3])$. Let $\sigma:[0, T] \times \mathbb{R} \rightarrow \mathbb{R}$. Assume that

(1) $\sigma \in C^{3}([0, T] \times \mathbb{R})$;

(2) there exists $C>0$ such that $|D \sigma(t, x)| \leq C$, where $D$ means an arbitrary operation of the differentiation which is allowed for $\sigma$ according to assumption (1);

(3) $|\sigma(r, 0)| \leq C$;

(4) $f \in W_{1}^{\alpha_{1}, \alpha_{2}}$ and $g \in W_{2}^{1-\alpha_{1}, 1-\alpha_{2}}$ for some $0<\alpha_{i}<\frac{1}{2}, i=1,2$.

Then the function $\sigma$ admits the following estimate:

$$
\|\sigma(\cdot, f(\cdot))\|_{1, \alpha_{1}, \alpha_{2}} \leq C_{\alpha_{1}, \alpha_{2}, T}(1+\|f\|)\left(1+\|f\|_{1, \alpha_{1}, \alpha_{2}}\right)^{2}
$$

where $C_{\alpha_{1}, \alpha_{2}, T}$ is a constant that depends on $\alpha_{1}, \alpha_{2}$, and $T$ only. The generalized Lebesgue-Stieltjes integral

$$
G_{t}^{(\sigma)}(f):=\int_{[0, t]} \sigma(s, f(s)) d g_{s}
$$

is well defined on $[0, T]$ and admits the following estimates:

$$
\left\|G^{(\sigma)}(f)\right\|_{1-\alpha_{1}, 1-\alpha_{2}} \leq C_{\alpha_{1}, \alpha_{2}, T} \Lambda_{\alpha_{1}, \alpha_{2}}(g)(1+\|f\|)\left(1+\|f\|_{1, \alpha_{1}, \alpha_{2}}\right)^{2}
$$


and

$$
\begin{aligned}
& \left\|G^{(\sigma)}(f)\right\|_{\alpha_{1}, \alpha_{2}, \lambda_{1}, \lambda_{2}} \\
& \quad \leq \frac{C_{\alpha_{1}, \alpha_{2}, T} \Lambda_{\alpha_{1}, \alpha_{2}}(g)}{\lambda_{1}^{1-2 \alpha_{1}} \lambda_{2}^{1-2 \alpha_{2}}}(1+\|f\|)^{2}\left(1+\|f\|_{\alpha_{1}, \alpha_{2}, \lambda_{1}, \lambda_{2}}+\|f\|_{\alpha_{1}, \alpha_{2}, \lambda_{1} / 2, \lambda_{2} / 2}^{2}\right) .
\end{aligned}
$$

In particular, $G^{(\sigma)}(f) \in \mathcal{H}^{1-\alpha_{1}, 1-\alpha_{2}}$. The constants $C_{\alpha_{1}, \alpha_{2}, T}$ in relations (5) and (6) depend on $\alpha_{1}, \alpha_{2}$, and $T$ and do not depend on $\lambda_{i}, i=1,2, f, h$, and $g$.

Remark 5. All the above estimates remain true if

$$
f(s)=g(s)=B_{s}, \quad s \geq 0,
$$

is a fractional Brownian field with Hurst indices $H_{i} \in\left(\frac{1}{2}, 1\right), i=1,2$.

Remark 6. Let the function $\sigma$ be bounded and

$$
\tilde{f}(x)=f(x)+C_{0},
$$

where $C_{0}$ is a constant. Then

$$
\begin{aligned}
& \left\|G^{(\sigma)}(\tilde{f})\right\|_{\alpha_{1}, \alpha_{2}, \lambda_{1}, \lambda_{2}} \\
& \quad \leq \frac{C_{\alpha_{1}, \alpha_{2}, T} \Lambda_{\alpha_{1}, \alpha_{2}}(g)}{\lambda_{1}^{1-2 \alpha_{1}} \lambda_{2}^{1-2 \alpha_{2}}}(1+\|f\|)^{2}\left(1+\|f\|_{\alpha_{1}, \alpha_{2}, \lambda_{1}, \lambda_{2}}+\|f\|_{\alpha_{1}, \alpha_{2}, \lambda_{1} / 2, \lambda_{2} / 2}^{2}\right) ;
\end{aligned}
$$

that is, the right hand side of the estimate does not depend on $C_{0}$.

Theorem 3. Let $\sigma:[0, T] \times \mathbb{R} \rightarrow \mathbb{R}$. Assume that

(1) $\sigma \in C^{3}[0, T] \times C^{5}(\mathbb{R})$;

(2) there exists $C>0$ such that

$$
|D \sigma(t, x)| \leq C,
$$

where $D$ means an arbitrary operation of the differentiation which is allowed for $\sigma$ according to assumption (1);

(3) $|\sigma(r, 0)| \leq C$;

(4) $f, h \in W_{1}^{\alpha_{1}, \alpha_{2}}$ and $g \in W_{2}^{1-\alpha_{1}, 1-\alpha_{2}}$ for some $0<\alpha_{i}<\frac{1}{2}, i=1,2$.

Then

$$
\begin{aligned}
&\left\|G^{(\sigma)}(f)-G^{(\sigma)}(h)\right\|_{\alpha_{1}, \alpha_{2}, \lambda_{1}, \lambda_{2}} \\
& \leq \frac{C_{\alpha_{1}, \alpha_{2}, T} \Lambda_{\alpha_{1}, \alpha_{2}}(g)}{\lambda_{1}^{1-2 \alpha_{1}} \lambda_{2}^{1-2 \alpha_{2}}}(1+\|f\|+\|h\|)^{2}\left(1+\|f\|_{1, \alpha_{1}, \alpha_{2}}+\|h\|_{1, \alpha_{1}, \alpha_{2}}\right)^{2} \\
& \times\left(\|f-h\|_{\alpha_{1}, \alpha_{2}, \lambda_{1}, \lambda_{2}}+\|f-h\|_{\alpha_{1}, \alpha_{2}, \lambda_{1} / 2, \lambda_{2} / 2}^{2}\right) .
\end{aligned}
$$

In particular, $G^{(\sigma)}(f), G^{(\sigma)}(h) \in \mathcal{H}^{1-\alpha_{1}, 1-\alpha_{2}}$. Here the constant $C_{\alpha_{1}, \alpha_{2}, T}$ depends on $\alpha_{1}$, $\alpha_{2}$, and $T$ and does not depend on $\lambda_{i}, i=1,2, f, h$, and $g$.

Proof. We derive from formulas (1) and (3) that

$$
\begin{aligned}
\left\|G_{t}^{(\sigma)}(f)-G_{t}^{(\sigma)}(h)\right\|_{\alpha_{1}, \alpha_{2}, \lambda_{1}, \lambda_{2}} & \\
\leq \Lambda_{\alpha_{1}, \alpha_{2}}(g) C_{\alpha_{1}, \alpha_{2}, T} \sup _{t \in[0, T]} e^{-\lambda_{1} t_{1}-\lambda_{2} t_{2}} & \int_{0}^{t_{1}} \int_{0}^{t_{2}}\left(r_{1}^{-\alpha_{1}}+\left(t_{1}-r_{1}\right)^{-2 \alpha_{1}}\right) \\
& \times\left(r_{2}^{-\alpha_{2}}+\left(t_{2}-r_{2}\right)^{-2 \alpha_{2}}\right) Y(r) d r_{1} d r_{2},
\end{aligned}
$$


where

$$
\begin{aligned}
Y(r)= & |\sigma(r, f(r))-\sigma(r, h(r))| \\
& +\int_{0}^{r_{1}} \frac{\left|\sigma(r, f(r))-\sigma\left(u, r_{2}, f\left(u, r_{2}\right)\right)-\sigma(r, h(r))+\sigma\left(u, r_{2}, h\left(u, r_{2}\right)\right)\right|}{\left(r_{1}-u\right)^{\alpha_{1}+1}} d u \\
& +\int_{0}^{r_{2}} \frac{\left|\sigma(r, f(r))-\sigma\left(r_{1}, v, f\left(r_{1}, v\right)\right)-\sigma(r, h(r))+\sigma\left(r_{1}, v, h\left(r_{1}, v\right)\right)\right|}{\left(r_{2}-v\right)^{\alpha_{2}+1}} d v \\
& +\int_{0}^{r_{1}} \int_{0}^{r_{2}} \frac{\left|\Delta_{u, v} \sigma(r, f(r))-\Delta_{u, v} \sigma(r, h(r))\right|}{\left(r_{1}-u\right)^{\alpha_{1}+1}\left(r_{2}-v\right)^{\alpha_{2}+1}} d u d v .
\end{aligned}
$$

Consider

$$
\begin{gathered}
\int_{0}^{t_{1}} \int_{0}^{t_{2}} e^{-\lambda_{1}\left(t_{1}-r_{1}\right)-\lambda_{2}\left(t_{2}-r_{2}\right)}\left(r_{1}^{-\alpha_{1}}+\left(t_{1}-r_{1}\right)^{-2 \alpha_{1}}\right)\left(r_{2}^{-\alpha_{2}}+\left(t_{2}-r_{2}\right)^{-2 \alpha_{2}}\right) d r \\
=\int_{0}^{t_{1}} \int_{0}^{t_{2}} e^{-\lambda_{1} x_{1}-\lambda_{2} x_{2}}\left(\left(t_{1}-x_{1}\right)^{-\alpha_{1}}+x_{1}^{-2 \alpha_{1}}\right)\left(\left(t_{2}-x_{2}\right)^{-\alpha_{2}}+x_{2}^{-2 \alpha_{2}}\right) d x \\
=\frac{1}{\lambda_{1} \lambda_{2}} \int_{0}^{\lambda_{1} t_{1}} \int_{0}^{\lambda_{2} t_{2}} e^{-y_{1}-y_{2}}\left(\lambda_{1}^{2 \alpha_{1}} y_{1}^{-2 \alpha_{1}}+\lambda_{1}^{\alpha_{1}}\left(\lambda_{1} t_{1}-y_{1}\right)^{-\alpha_{1}}\right) \\
\quad \times\left(\lambda_{2}^{2 \alpha_{2}} y_{2}^{-2 \alpha_{2}}+\lambda_{2}^{\alpha_{2}}\left(\lambda_{2} t_{2}-y_{2}\right)^{-\alpha_{2}}\right) d y \\
\leq \lambda_{1}^{2 \alpha_{1}-1} \lambda_{2}^{2 \alpha_{2}-1}\left(\int_{0}^{\infty} e^{-y_{1}} y_{1}^{-2 \alpha_{1}} d y_{1}+\sup _{z_{1}>0} \int_{0}^{z_{1}} e^{-y_{1}}\left(z_{1}-y_{1}\right)^{-\alpha_{1}} d y_{1}\right) \\
\quad \times\left(\int_{0}^{\infty} e^{-y_{2}} y_{2}^{-2 \alpha_{2}} d y_{2}+\sup _{z_{2}>0}^{z_{2}} e_{0}^{-y_{2}}\left(z_{2}-y_{2}\right)^{-\alpha_{2}} d y_{2}\right) \\
:=\lambda_{1}^{2 \alpha_{1}-1} \lambda_{2}^{2 \alpha_{2}-1} C_{\alpha_{1}, \alpha_{2}},
\end{gathered}
$$

where

$$
C_{\alpha_{1}, \alpha_{2}} \leq\left(\left(1-2 \alpha_{1}\right)^{-1}+4\right)\left(\left(1-2 \alpha_{2}\right)^{-1}+4\right) .
$$

We estimate the first term on the right hand side of (9) by using the Lagrange theorem:

$$
|\sigma(r, f(r))-\sigma(r, h(r))| \leq C|q(r)|,
$$

where $q(\cdot):=f(\cdot)-h(\cdot)$.

Consider the numerator of the fourth term in (9). The other numerators are estimated similarly. We have

$$
\begin{aligned}
& \left|\Delta_{s} \sigma(r, f(r))-\Delta_{s} \sigma(r, h(r))\right| \\
& =\mid \sigma(r, f(r))-\sigma\left(r_{1}, s_{2}, f\left(r_{1} s_{2}\right)\right)-\sigma\left(s_{1}, r_{2}, f\left(s_{1}, r_{2}\right)\right) \\
& \quad+\sigma(s, f(s))-\sigma(r, h(r))+\sigma\left(r_{1}, s_{2}, h\left(r_{1}, s_{2}\right)\right)+\sigma\left(s_{1}, r_{2}, h\left(s_{1}, r_{2}\right)\right)-\sigma(s, f(s)) \mid .
\end{aligned}
$$

Now we apply the Taylor formula obtained in [4]:

$$
f(b)-f(a)=f^{\prime}(a)(b-a)+(b-a)^{2} \int_{0}^{1} \alpha f^{\prime \prime}(\alpha a+(1-\alpha) b) d \alpha .
$$

In what follows we use the following notation:

$$
\begin{aligned}
\sigma(u, f(u))-\sigma(u, h(u)) & =\sigma_{3}^{\prime}(u, h(u)) q(u)+(q(u))^{2} \int_{0}^{1} \alpha \sigma_{33}^{\prime \prime}(u, \alpha h(u)+(1-\alpha) f(u)) d \alpha \\
& :=z^{1}(u)+z^{2}(u)
\end{aligned}
$$

that is,

$$
\sigma_{3}^{\prime}(u, h(u)) q(u):=z^{1}(u)
$$


and

$$
\begin{gathered}
(q(u))^{2} \int_{0}^{1} \alpha \sigma_{33}^{\prime \prime}(u, \alpha h(u)+(1-\alpha) f(u)) d \alpha:=z^{2}(u) \\
u=r, s,\left(r_{1}, s_{2}\right),\left(s_{1}, r_{2}\right)
\end{gathered}
$$

We consider the sums $S^{1}:=\Delta_{s} z^{1}(r)$ and $S^{2}:=\Delta_{s} z^{2}(r)$ separately.

Note that

$$
\left|\Delta_{s} \sigma(r, f(r))-\Delta_{s} \sigma(r, h(r))\right|=\left|S^{1}+S^{2}\right|=: S .
$$

We have

$$
\begin{aligned}
S^{1}= & \Delta_{s} z^{1}(r) \\
= & z^{1}(r)-z^{1}\left(r_{1}, s_{2}\right)-z^{1}\left(s_{1}, r_{2}\right)+z^{1}(s) \\
= & \sigma_{3}^{\prime}(r, h(r)) q(r)-\sigma_{3}^{\prime}\left(r_{1}, s_{2}, h\left(r_{1}, s_{2}\right)\right) q\left(r_{1}, s_{2}\right)-\sigma_{3}^{\prime}\left(s_{1}, r_{2}, h\left(s_{1}, r_{2}\right)\right) q\left(s_{1}, r_{2}\right) \\
& +\sigma_{3}^{\prime}(s, h(s)) q(s) \\
= & \Delta_{s} \sigma_{3}^{\prime}(r, h(r)) q(r)+\left[\sigma_{3}^{\prime}(s, h(s))-\sigma_{3}^{\prime}\left(r_{1}, s_{2}, h\left(r_{1}, s_{2}\right)\right)\right]\left[q\left(r_{1}, s_{2}\right)-q(r)\right] \\
& +\left[\sigma_{3}^{\prime}(s, h(s))-\sigma_{3}^{\prime}\left(s_{1}, r_{2}, h\left(s_{1}, r_{2}\right)\right)\right]\left[q\left(s_{1}, r_{2}\right)-q(r)\right]+\sigma_{3}^{\prime}(s, h(s)) \Delta_{s} q(r) \\
= & I_{1}+I_{2}+I_{3}+I_{4} .
\end{aligned}
$$

By the Lagrange theorem and assumption (2) of Theorem 3 we get

$$
\begin{aligned}
& \left|I_{2}\right| \leq C_{\alpha_{1}, \alpha_{2}, T}\left[\left(r_{1}-s_{1}\right)\left|q\left(r_{1}, s_{2}\right)-q(r)\right|+\left|h\left(r_{1}, s_{2}\right)-h(s)\right|\left|q\left(r_{1}, s_{2}\right)-q(r)\right|\right] \\
& \leq C_{\alpha_{1}, \alpha_{2}, T}\left[\left(r_{1}-s_{1}\right)\left|q\left(r_{1}, s_{2}\right)-q(r)\right|+\left|\Delta_{s} h(r)\right|\left|q\left(r_{1}, s_{2}\right)-q(r)\right|\right. \\
& \left.+\left|h(r)-h\left(s_{1}, r_{2}\right)\right|\left|q\left(r_{1}, s_{2}\right)-q(r)\right|\right], \\
& \left|I_{3}\right| \leq C_{\alpha_{1}, \alpha_{2}, T}\left[\left(r_{2}-s_{2}\right)\left|q\left(s_{1}, r_{2}\right)-q(r)\right|+\left|\Delta_{s} h(r)\right|\left|q\left(s_{1}, r_{2}\right)-q(r)\right|\right. \\
& \left.+\left|h(r)-h\left(r_{1}, s_{2}\right)\right|\left|q\left(s_{1}, r_{2}\right)-q(r)\right|\right], \\
& \left|I_{4}\right| \leq C_{\alpha_{1}, \alpha_{2}, T}\left|\Delta_{s} q(r)\right| \text {, } \\
& \left|\Delta_{s} \sigma_{3}^{\prime}(r, h(r))\right|=\mid \sigma_{3}^{\prime}(r, h(r))-\sigma_{3}^{\prime}\left(s_{1}, r_{2}, h\left(s_{1}, r_{2}\right)\right) \\
& -\sigma_{3}^{\prime}\left(r_{1}, s_{2}, h\left(r_{1}, s_{2}\right)\right)+\sigma_{3}^{\prime}(s, h(s)) \\
& =\mid \sigma_{3}^{\prime}(r, h(r))-\sigma_{3}^{\prime}\left(s_{1}, r_{2}, h(r)\right)+\sigma_{3}^{\prime}\left(s_{1}, r_{2}, h(r)\right) \\
& -\sigma_{3}^{\prime}\left(s_{1}, r_{2}, h\left(s_{1}, r_{2}\right)\right)-\left(\sigma_{3}^{\prime}\left(r_{1}, s_{2}, h\left(r_{1}, s_{2}\right)\right)-\sigma_{3}^{\prime}\left(s, h\left(r_{1}, s_{2}\right)\right)\right) \\
& -\left(\sigma_{3}^{\prime}\left(s, h\left(r_{1}, s_{2}\right)\right)-\sigma_{3}^{\prime}(s, h(s))\right) \\
& =\left|P_{1}+P_{2}-P_{3}-P_{4}\right| .
\end{aligned}
$$

Applying the Taylor formula (12) we obtain

$$
\begin{aligned}
P_{1}= & \sigma_{13}^{\prime \prime}\left(s_{1}, r_{2}, h(r)\right)\left(r_{1}-s_{1}\right) \\
& +\left(r_{1}-s_{1}\right)^{2} \int_{0}^{1} \alpha \sigma_{113}^{(3)}\left(\alpha s_{1}+(1-\alpha) r_{1}, r_{2}, h(r)\right) d \alpha,
\end{aligned}
$$




$$
\begin{aligned}
P_{2}= & \sigma_{33}^{\prime \prime}\left(s_{1}, r_{2}, h\left(s_{1}, r_{2}\right)\right)\left(h(r)-h\left(s_{1}, r_{2}\right)\right) \\
& +\left(h(r)-h\left(s_{1}, r_{2}\right)\right)^{2} \int_{0}^{1} \alpha \sigma_{333}^{(3)}\left(s_{1}, r_{2}, \alpha h\left(s_{1}, r_{2}\right)+(1-\alpha) h(r)\right) d \alpha, \\
P_{3}= & \sigma_{13}^{\prime \prime}\left(s, h\left(r_{1}, s_{2}\right)\right)\left(r_{1}-s_{1}\right) \\
& +\left(r_{1}-s_{1}\right)^{2} \int_{0}^{1} \alpha \sigma_{113}^{(3)}\left(\alpha s_{1}+(1-\alpha) r_{1}, s_{2}, h\left(r_{1}, s_{2}\right)\right) d \alpha, \\
P_{4}= & \sigma_{33}^{\prime \prime}(s, h(s))\left(h\left(r_{1}, s_{2}\right)-h(s)\right) \\
& +\left(h\left(r_{1}, s_{2}\right)-h(s)\right)^{2} \int_{0}^{1} \alpha \sigma_{333}^{(3)}\left(s, \alpha h(s)+(1-\alpha) h\left(r_{1}, s_{2}\right)\right) d \alpha .
\end{aligned}
$$

Now we estimate these differences separately with the help of the Lagrange theorem. In doing so we use the derivatives of $\sigma$ up to the fourth order:

$$
\begin{aligned}
& \left|P_{1}-P_{3}\right|=\left|\left(\sigma_{13}^{\prime \prime}\left(s_{1}, r_{2}, h(r)\right)-\sigma_{13}^{\prime \prime}\left(s, h\left(r_{1}, s_{2}\right)\right)\right)\left(r_{1}-s_{1}\right)\right| \\
& +\left(r_{1}-s_{1}\right)^{2} \mid \int_{0}^{1} \alpha \sigma_{113}^{(3)}\left(\alpha s_{1}+(1-\alpha) r_{1}, r_{2}, h(r)\right) d \alpha \\
& -\int_{0}^{1} \alpha \sigma_{113}^{(3)}\left(\alpha s_{1}+(1-\alpha) r_{1}, s_{2}, h\left(r_{1}, s_{2}\right)\right) d \alpha \mid \\
& \leq C_{\alpha_{1}, \alpha_{2}, T}\left(\left(r_{1}-s_{1}\right)\left(r_{2}-s_{2}\right)+\left|h(r)-h\left(r_{1}, s_{2}\right)\right|\left(r_{1}-s_{1}\right)\right. \\
& \left.+\left(r_{1}-s_{1}\right)\left(r_{2}-s_{2}\right)+\left|h(r)-h\left(r_{1}, s_{2}\right)\right|\left(r_{1}-s_{1}\right)\right) \\
& \leq C_{\alpha_{1}, \alpha_{2}, T}\left(\left(r_{1}-s_{1}\right)\left(r_{2}-s_{2}\right)+\left|h(r)-h\left(r_{1}, s_{2}\right)\right|\left(r_{1}-s_{1}\right)\right) \text {, } \\
& \left|P_{2}-P_{4}\right|=\mid \sigma_{33}^{\prime \prime}\left(s_{1}, r_{2}, h\left(s_{1}, r_{2}\right)\right)\left(h(r)-h\left(s_{1}, r_{2}\right)\right) \\
& +\left(h(r)-h\left(s_{1}, r_{2}\right)\right)^{2} \int_{0}^{1} \alpha \sigma_{333}^{(3)}\left(s_{1}, r_{2}, \alpha h\left(s_{1}, r_{2}\right)+(1-\alpha) h(r)\right) d \alpha \\
& -\sigma_{33}^{\prime \prime}(s, h(s))\left(h\left(r_{1}, s_{2}\right)-h(s)\right) \\
& -\left(h\left(r_{1}, s_{2}\right)-h(s)\right)^{2} \int_{0}^{1} \alpha \sigma_{333}^{(3)}\left(s, \alpha h(s)+(1-\alpha) h\left(r_{1}, s_{2}\right)\right) d \alpha \mid \\
& =\mid \Delta_{s} h(r) \sigma_{33}^{\prime \prime}(s, h(s)) \\
& +\left(\sigma_{33}^{\prime \prime}\left(s_{1}, r_{2}, h\left(s_{1}, r_{2}\right)\right)-\sigma_{33}^{\prime \prime}(s, h(s))\right)\left(h(r)-h\left(s_{1}, r_{2}\right)\right) \\
& +\left(h(r)-h\left(s_{1}, r_{2}\right)\right)^{2}\left[\int_{0}^{1} \alpha \sigma_{333}^{(3)}\left(s_{1}, r_{2}, \alpha h\left(s_{1}, r_{2}\right)+(1-\alpha) h(r)\right) d \alpha\right. \\
& \left.-\int_{0}^{1} \alpha \sigma_{333}^{(3)}\left(s, \alpha h(s)+(1-\alpha) h\left(r_{1}, s_{2}\right)\right) d \alpha\right] \\
& +\left(\left(h(r)-h\left(s_{1}, r_{2}\right)\right)^{2}-\left(h\left(r_{1}, s_{2}\right)-h(s)\right)^{2}\right) \\
& \times \int_{0}^{1} \alpha \sigma_{333}^{(3)}\left(s, \alpha h(s)+(1-\alpha) h\left(r_{1}, s_{2}\right)\right) d \alpha \mid \\
& \leq C_{\alpha_{1}, \alpha_{2}, T}\left(\left|\Delta_{s} h(r)\right|+\left(r_{2}-s_{2}\right)\left|h(r)-h\left(s_{1}, r_{2}\right)\right|\right. \\
& +\left|h\left(s_{1}, r_{2}\right)-h(s)\right|\left|h(r)-h\left(s_{1}, r_{2}\right)\right| \\
& +\|h\|\left(r_{2}-s_{2}\right)\left|h(r)-h\left(s_{1}, r_{2}\right)\right|
\end{aligned}
$$




$$
\begin{aligned}
&+\|h\|\left|h\left(s_{1}, r_{2}\right)-h(s)\right|\left|h(r)-h\left(s_{1}, r_{2}\right)\right| \\
&+\|h\|\left|h(r)-h\left(s_{1}, r_{2}\right)\right|\left|h(r)-h\left(r_{1}, s_{2}\right)\right| \\
&\left.+\left|\Delta_{s} h(r)\right|\left|h(r)-h\left(s_{1}, r_{2}\right)\right|+\left|\Delta_{s} h(r)\right|\left|h\left(r_{1}, s_{2}\right)-h(s)\right|\right) \\
& \leq C_{\alpha_{1}, \alpha_{2}, T}\left(\left|\Delta_{s} h(r)\right|+\left(r_{2}-s_{2}\right)\left|h(r)-h\left(s_{1}, r_{2}\right)\right|\right. \\
&+\left|h\left(s_{1}, r_{2}\right)-h(s)\right|\left|h(r)-h\left(s_{1}, r_{2}\right)\right| \\
&+\|h\|\left(r_{2}-s_{2}\right)\left|h(r)-h\left(s_{1}, r_{2}\right)\right| \\
&+\|h\|\left|h\left(s_{1}, r_{2}\right)-h(s)\right|\left|h(r)-h\left(s_{1}, r_{2}\right)\right| \\
&\left.+\|h\|\left|h(r)-h\left(s_{1}, r_{2}\right)\right|\left|h(r)-h\left(r_{1}, s_{2}\right)\right|+\|h\|\left|\Delta_{s} h(r)\right|\right) \\
& \leq C \alpha_{1}, \alpha_{2}, T\left((1+\|h\|)\left(r_{2}-s_{2}\right)\left|h(r)-h\left(s_{1}, r_{2}\right)\right|+(1+\|h\|)^{2}\left|\Delta_{s} h(r)\right|\right. \\
&\left.+(1+\|h\|)\left|h(r)-h\left(s_{1}, r_{2}\right) \| h(r)-h\left(r_{1}, s_{2}\right)\right|\right) .
\end{aligned}
$$

Substituting (15) and (16) into (14), we get

$$
\begin{aligned}
\left|\Delta_{s} \sigma_{3}^{\prime}(r, h(r))\right| \leq C_{\alpha_{1}, \alpha_{2}, T}( & \left(r_{1}-s_{1}\right)\left(r_{2}-s_{2}\right)+\left(r_{1}-s_{1}\right)\left|h(r)-h\left(r_{1}, s_{2}\right)\right| \\
& +(1+\|h\|)\left(r_{2}-s_{2}\right)\left|h(r)-h\left(s_{1}, r_{2}\right)\right| \\
& +(1+\|h\|)^{2}\left|\Delta_{s} h(r)\right| \\
& \left.+(1+\|h\|)\left|h(r)-h\left(s_{1}, r_{2}\right)\right| \cdot\left|h(r)-h\left(r_{1}, s_{2}\right)\right|\right) .
\end{aligned}
$$

Thus

$$
\begin{aligned}
\left|\Delta_{s} z^{1}(r)\right| \leq C_{\alpha_{1}, \alpha_{2}, T}( & \left(r_{1}-s_{1}\right)\left(r_{2}-s_{2}\right)|q(r)|+\left(r_{1}-s_{1}\right)\left|h(r)-h\left(r_{1}, s_{2}\right)\right||q(r)| \\
& +(1+\|h\|)\left(r_{2}-s_{2}\right)\left|h(r)-h\left(s_{1}, r_{2}\right)\right||q(r)| \\
& +(1+\|h\|)^{2}\left|\Delta_{s} h(r)\right||q(r)| \\
& +(1+\|h\|)\left|h(r)-h\left(s_{1}, r_{2}\right)\right|\left|h(r)-h\left(r_{1}, s_{2}\right)\right||q(r)| \\
& +\left(r_{1}-s_{1}\right)\left|q\left(r_{1}, s_{2}\right)-q(r)\right|+\left|\Delta_{s} h(r)\right|\left|q\left(r_{1}, s_{2}\right)-q(r)\right| \\
& +\left|h(r)-h\left(s_{1}, r_{2}\right)\right|\left|q\left(r_{1}, s_{2}\right)-q(r)\right| \\
& +\left(r_{2}-s_{2}\right)\left|q\left(s_{1}, r_{2}\right)-q(r)\right|+\left|\Delta_{s} h(r)\right|\left|q\left(s_{1}, r_{2}\right)-q(r)\right| \\
& \left.\quad+\left|h(r)-h\left(r_{1}, s_{2}\right)\right|\left|q\left(s_{1}, r_{2}\right)-q(r)\right|+\left|\Delta_{s} q(r)\right|\right) .
\end{aligned}
$$

Then

$$
\begin{aligned}
S^{2}= & \Delta_{s} z^{2}(r) \\
= & z^{2}(r)-z^{2}\left(r_{1}, s_{2}\right)-z^{2}\left(s_{1}, r_{2}\right)+z^{2}(s) \\
= & (q(r))^{2} \int_{0}^{1} \alpha \sigma_{33}^{\prime \prime}(r, \alpha h(r)+(1-\alpha) f(r)) d \alpha \\
& -\left(q\left(r_{1}, s_{2}\right)\right)^{2} \int_{0}^{1} \alpha \sigma_{33}^{\prime \prime}\left(r_{1}, s_{2}, \alpha h\left(r_{1}, s_{2}\right)+(1-\alpha) f\left(r_{1}, s_{2}\right)\right) d \alpha \\
& -\left(q\left(s_{1}, r_{2}\right)\right)^{2} \int_{0}^{1} \alpha \sigma_{33}^{\prime \prime}\left(s_{1}, r_{2}, \alpha h\left(s_{1}, r_{2}\right)+(1-\alpha) f\left(s_{1}, r_{2}\right)\right) d \alpha \\
& +(q(s))^{2} \int_{0}^{1} \alpha \sigma_{33}^{\prime \prime}(s, \alpha h(s)+(1-\alpha) f(s)) d \alpha
\end{aligned}
$$




$$
\begin{aligned}
&=\int_{0}^{1} \alpha\left[(q(r))^{2} \sigma_{33}^{\prime \prime}(r, \alpha h(r)\right.+(1-\alpha) f(r)) \\
&-\left(q\left(r_{1}, s_{2}\right)\right)^{2} \sigma_{33}^{\prime \prime}\left(r_{1}, s_{2}, \alpha h\left(r_{1}, s_{2}\right)+(1-\alpha) f\left(r_{1}, s_{2}\right)\right) \\
&-\left(q\left(s_{1}, r_{2}\right)\right)^{2} \sigma_{33}^{\prime \prime}\left(s_{1}, r_{2}, \alpha h\left(s_{1}, r_{2}\right)+(1-\alpha) f\left(s_{1}, r_{2}\right)\right) \\
&\left.+(q(s))^{2} \sigma_{33}^{\prime \prime}(s, \alpha h(s)+(1-\alpha) f(s))\right] d \alpha .
\end{aligned}
$$

One can apply estimate (17) to the expression written in the square brackets in (18) where $q^{2}(\cdot)$ and $\left.(\alpha h(\cdot)+(1-\alpha) f(\cdot))\right)$ are substituted for $q(\cdot)$ and $h(\cdot)$, respectively. Using the existence of the derivatives of $\sigma$ up to the fifth order we obtain

$$
\begin{aligned}
\left|\Delta_{s} z^{2}(r)\right| \leq C_{\alpha_{1}, \alpha_{2}, T} & \left(r_{1}-s_{1}\right)\left(r_{2}-s_{2}\right)\left|q^{2}(r)\right|+\left(r_{1}-s_{1}\right)\left|h(r)-h\left(r_{1}, s_{2}\right)\right|\left|q^{2}(r)\right| \\
& +\left(r_{1}-s_{1}\right)\left|f(r)-f\left(r_{1}, s_{2}\right)\right|\left|q^{2}(r)\right| \\
& +(1+\|h\|+\|f\|)\left(r_{2}-s_{2}\right)\left|h(r)-h\left(s_{1}, r_{2}\right)\right|\left|q^{2}(r)\right| \\
& +(1+\|h\|+\|f\|)\left(r_{2}-s_{2}\right)\left|f(r)-f\left(s_{1}, r_{2}\right)\right|\left|q^{2}(r)\right| \\
& +(1+\|h\|+\|f\|)^{2}\left|\Delta_{s} h(r)\right|\left|q^{2}(r)\right| \\
& +(1+\|h\|+\|f\|)^{2}\left|\Delta_{s} f(r)\right|\left|q^{2}(r)\right| \\
& +(1+\|h\|+\|f\|)\left|h(r)-h\left(s_{1}, r_{2}\right)\right|\left|h(r)-h\left(r_{1}, s_{2}\right)\right|\left|q^{2}(r)\right| \\
& +(1+\|h\|+\|f\|)\left|h(r)-h\left(s_{1}, r_{2}\right)\right|\left|f(r)-f\left(r_{1}, s_{2}\right)\right|\left|q^{2}(r)\right| \\
& +(1+\|h\|+\|f\|)\left|f(r)-f\left(s_{1}, r_{2}\right)\right|\left|h(r)-h\left(r_{1}, s_{2}\right)\right|\left|q^{2}(r)\right| \\
& +(1+\|h\|+\|f\|)\left|f(r)-f\left(s_{1}, r_{2}\right)\right|\left|f(r)-f\left(r_{1}, s_{2}\right)\right|\left|q^{2}(r)\right| \\
& +\left(r_{1}-s_{1}\right)\left|q^{2}\left(r_{1}, s_{2}\right)-q^{2}(r)\right|+\left|\Delta_{s} h(r)\right|\left|q^{2}\left(r_{1}, s_{2}\right)-q^{2}(r)\right| \\
& +\left|\Delta_{s} f(r)\right|\left|q^{2}\left(r_{1}, s_{2}\right)-q^{2}(r)\right| \\
& +\left|h(r)-h\left(s_{1}, r_{2}\right)\right|\left|q^{2}\left(r_{1}, s_{2}\right)-q^{2}(r)\right| \\
& +\left|f(r)-f\left(s_{1}, r_{2}\right)\right|\left|q^{2}\left(r_{1}, s_{2}\right)-q^{2}(r)\right| \\
& +\left(r_{2}-s_{2}\right)\left|q^{2}\left(s_{1}, r_{2}\right)-q^{2}(r)\right|+\left|\Delta_{s} h(r)\right|\left|q^{2}\left(s_{1}, r_{2}\right)-q^{2}(r)\right| \\
& +\left|\Delta_{s} f(r)\right|\left|q^{2}\left(s_{1}, r_{2}\right)-q^{2}(r)\right| \\
& +\left|h(r)-h\left(r_{1}, s_{2}\right)\right|\left|q^{2}\left(s_{1}, r_{2}\right)-q^{2}(r)\right| \\
& \left.\quad+\left|f(r)-f\left(r_{1}, s_{2}\right)\right|\left|q^{2}\left(s_{1}, r_{2}\right)-q^{2}(r)\right|+\left|\Delta_{s} q^{2}(r)\right|\right) . \\
&
\end{aligned}
$$

The last term in (19) is estimated as follows:

$$
\begin{aligned}
\left|\Delta_{s} q^{2}(r)\right|= & \left|q^{2}(r)-q^{2}\left(r_{1}, s_{2}\right)-q^{2}\left(s_{1}, r_{2}\right)+q^{2}(s)\right| \\
= & \left|\left(q(r)-q\left(r_{1}, s_{2}\right)\right)\left(q(r)+q\left(r_{1}, s_{2}\right)\right)-\left(q\left(s_{1}, r_{2}\right)-q(s)\right)\left(q\left(s_{1}, r_{2}\right)+q(s)\right)\right| \\
\leq & \left|\Delta_{s} q(r)\right|\left|q(r)-q\left(r_{1}, s_{2}\right)\right| \\
& +\left|q\left(s_{1}, r_{2}\right)+q(s)\right|\left|q(r)+q\left(r_{1}, s_{2}\right)-q\left(s_{1}, r_{2}\right)-q(s)\right| \\
\leq & 2\left(\|q\|\left|\Delta_{s} q(r)\right|+\left|q(r)-q\left(r_{1}, s_{2}\right)\right|\left|q(r)-q\left(s_{1}, r_{2}\right)\right|\right), \\
& \left|q^{2}\left(s_{1}, r_{2}\right)-q^{2}(r)\right| \leq 2\|q\||| q\left(s_{1}, r_{2}\right)-q(r) \mid .
\end{aligned}
$$


Substitute (17) and (19) into (13):

$$
\begin{aligned}
S \leq C_{\alpha_{1}, \alpha_{2}, T} & (1+\|q\|)\left(r_{1}-s_{1}\right)\left(r_{2}-s_{2}\right)|q(r)| \\
& +(1+\|q\|)\left(r_{1}-s_{1}\right)\left|h(r)-h\left(r_{1}, s_{2}\right)\right| \cdot|q(r)| \\
& +\|q\|\left(r_{1}-s_{1}\right)\left|f(r)-f\left(r_{1}, s_{2}\right)\right| \cdot|q(r)| \\
& +(1+\|q\|)(1+\|h\|+\|f\|)\left(r_{2}-s_{2}\right)\left|h(r)-h\left(s_{1}, r_{2}\right)\right| \cdot|q(r)| \\
& +\|q\|(1+\|h\|+\|f\|)\left(r_{2}-s_{2}\right)\left|f(r)-f\left(s_{1}, r_{2}\right)\right| \cdot|q(r)| \\
& +(1+\|q\|)(1+\|h\|+\|f\|)^{2}\left|\Delta_{s} h(r)\right| \cdot|q(r)| \\
& +\|q\|(1+\|h\|+\|f\|)^{2}\left|\Delta_{s} f(r)\right| \cdot|q(r)| \\
& +(1+\|q\|)(1+\|h\|+\|f\|) \\
& \times\left|h(r)-h\left(s_{1}, r_{2}\right)\right| \cdot\left|h(r)-h\left(r_{1}, s_{2}\right)\right| \cdot|q(r)| \\
& +\|q\|(1+\|h\|+\|f\|)\left|h(r)-h\left(s_{1}, r_{2}\right)\right| \cdot\left|f(r)-f\left(r_{1}, s_{2}\right)\right| \cdot|q(r)| \\
& +\|q\|(1+\|h\|+\|f\|)\left|f(r)-f\left(s_{1}, r_{2}\right)\right| \cdot\left|h(r)-h\left(r_{1}, s_{2}\right)\right| \cdot|q(r)| \\
& +\|q\|(1+\|h\|+\|f\|)\left|f(r)-f\left(s_{1}, r_{2}\right)\right| \cdot\left|f(r)-f\left(r_{1}, s_{2}\right)\right| \cdot|q(r)| \\
& +(1+\|q\|)\left(r_{1}-s_{1}\right)\left|q\left(r_{1}, s_{2}\right)-q(r)\right| \\
& +(1+\|q\|)\left|\Delta_{s} h(r)\right| \cdot\left|q\left(r_{1}, s_{2}\right)-q(r)\right| \\
& +\|q\|\left|\Delta_{s} f(r)\right| \cdot\left|q\left(r_{1}, s_{2}\right)-q(r)\right| \\
& +(1+\|q\|)\left|h(r)-h\left(s_{1}, r_{2}\right)\right| \cdot\left|q\left(r_{1}, s_{2}\right)-q(r)\right| \\
& +\|q\|\left|f(r)-f\left(s_{1}, r_{2}\right)\right| \cdot\left|q\left(r_{1}, s_{2}\right)-q(r)\right| \\
& +(1+\|q\|)\left(r_{2}-s_{2}\right)\left|q\left(s_{1}, r_{2}\right)-q(r)\right| \\
& +(1+\|q\|)\left|\Delta_{s} h(r)\right| \cdot\left|q\left(s_{1}, r_{2}\right)-q(r)\right| \\
& +\|q\|\left|\Delta_{s} f(r)\right| \cdot\left|q\left(s_{1}, r_{2}\right)-q(r)\right| \\
& +(1+\|q\|)\left|h(r)-h\left(r_{1}, s_{2}\right)\right| \cdot\left|q\left(s_{1}, r_{2}\right)-q(r)\right| \\
& +\|q\|\left|f(r)-f\left(r_{1}, s_{2}\right)\right| \cdot\left|q\left(s_{1}, r_{2}\right)-q(r)\right| \\
& \left.\quad+(1+\|q\|)\left|\Delta_{s} q(r)\right|+\left|q(r)-q\left(r_{1}, s_{2}\right)\right| \cdot\left|q(r)-q\left(s_{1}, r_{2}\right)\right|\right) .
\end{aligned}
$$

Using estimates (10), (11), and (20) in inequality (8) we establish bound (7). This completes the proof.

Now we obtain analogous bounds for the usual Lebesgue integral. The proof is similar to the above one; however, it is much simpler and we omit it. Let

$$
F_{t}(f)=\int_{[0, t]} f(s) d s \quad \text { and } \quad F_{t}^{(b)}(f)=\int_{[0, t]} b(s, f(s)) d s
$$

where $s=\left(s_{1}, s_{2}\right)$ and $t=\left(t_{1}, t_{2}\right), s, t \in[0, T]$.

Theorem 4. Let $0<\alpha_{i}<\frac{1}{2}, i=1,2$, and let $f:[0, T] \rightarrow \mathbb{R}$ be a measurable function. Assume that

$$
\sup _{t \in[0, T]} \int_{0}^{t} \frac{|f(s)|}{\left(t_{1}-s_{1}\right)^{\alpha_{1}}\left(t_{2}-s_{2}\right)^{\alpha_{2}}} d s_{1} d s_{2}<\infty
$$


(this inequality holds, in particular, if $\|f\|<\infty)$. Then $F .(f) \in W_{1}^{\alpha_{1}, \alpha_{2}}$ and

$$
\begin{aligned}
\left|F_{t}(f)\right| & +\int_{0}^{t_{1}} \frac{\left|F_{t}(f)-F_{s_{1}, t_{2}}(f)\right|}{\left(t_{1}-s_{1}\right)^{\alpha_{1}+1}} d s_{1}+\int_{0}^{t_{2}} \frac{\left|F_{t}(f)-F_{t_{1}, s_{2}}(f)\right|}{\left(t_{2}-s_{2}\right)^{\alpha_{2}+1}} d s_{2} \\
& +\int_{[0, t]} \frac{\left|\Delta_{s} F_{t}(f)\right|}{\left(t_{1}-s_{1}\right)^{\alpha_{1}+1}\left(t_{2}-s_{2}\right)^{\alpha_{2}+1}} d s \\
\leq & C_{\alpha_{1}, \alpha_{2}, T} \int_{[0, t]} \frac{|f(s)|}{\left(t_{1}-s_{1}\right)^{\alpha_{1}}\left(t_{2}-s_{2}\right)^{\alpha_{2}}} d s
\end{aligned}
$$

for all $0 \leq t \leq T$.

If $f \in W_{1}^{\alpha_{1}, \alpha_{2}}$, then $F .(f) \in C^{1}[0, T]$ and thus

$$
\left|\Delta_{s} F_{t}(f)\right|=\left|\int_{s_{1}}^{t_{1}} \int_{s_{2}}^{t_{2}} f d r\right| \leq\left(t_{1}-s_{1}\right)\left(t_{2}-s_{2}\right)\|f\|
$$

and

$$
\|F(f)\|_{1, \alpha_{1}, \alpha_{2}}=\left\|\int_{[0, \cdot]} f d s\right\|_{1, \alpha_{1}, \alpha_{2}} \leq C_{\alpha_{1}, \alpha_{2}, T}^{\prime}\|f\|
$$

where $C_{\alpha_{1}, \alpha_{2}, T}$ and $C_{\alpha_{1}, \alpha_{2}, T}^{\prime}$ are some constants that depend on $\alpha_{1}, \alpha_{2}$, and $T$ only.

Theorem 5. Assume that $|b(t, x)| \leq C_{0}(1+|x|)$ for all $x \in \mathbb{R}$ and $t \in[0, T]$. If $f \in W_{1}^{\alpha_{1}, \alpha_{2}}$, then $F^{(b)}(f)=\int_{[0, \cdot]} b(s, f(s)) d s \in C^{1}([0, T])$ and

$$
\left\|F^{(b)}(f)\right\|_{\alpha_{1}, \alpha_{2}, \lambda_{1}, \lambda_{2}} \leq \frac{C_{\alpha_{1}, \alpha_{2}, T}}{\lambda_{1}^{1-\alpha_{1}} \lambda_{2}^{1-\alpha_{2}}}\left(1+\|f\|_{\alpha_{1}, \alpha_{2}, \lambda_{1}, \lambda_{2}}\right)
$$

for all $\lambda_{i} \geq 1, i=1,2$, where $C_{\alpha_{1}, \alpha_{2}, T}$ is a positive constant that depends on $\alpha_{1}, \alpha_{2}$, and $T$ only.

Remark 7. If $b(t, x)$ is bounded, then the result discussed in Remark 6 holds.

Theorem 6. If $f, h \in W_{1}^{\alpha_{1}, \alpha_{2}}$ are such that $\|f\| \leq N$ and $\|h\| \leq N$, then

$$
\left\|F^{(b)}(f)-F^{(b)}(h)\right\|_{\alpha_{1}, \alpha_{2}, \lambda_{1}, \lambda_{2}} \leq \frac{C_{\alpha_{1}, \alpha_{2}, T, N}}{\lambda_{1}^{1-\alpha_{1}} \lambda_{2}^{1-\alpha_{2}}}\|f-h\|_{\alpha_{1}, \alpha_{2}, \lambda_{1}, \lambda_{2}}
$$

for all $\lambda_{i} \geq 1$, where $C_{\alpha_{1}, \alpha_{2}, T, N}$ is some constant that depends on $\alpha_{1}, \alpha_{2}, T$, and $N$ only.

3. The EXistence And uniqueness of a solution of a stochastic DIFFERENTIAL EQUATION WITH RESPECT TO A FRACTIONAL BROWNIAN FIELD

The notion of a fractional Brownian field on the plane can be introduced in several ways. We consider the following definition of a fractional Brownian field that preserves the Brownian motion properties coordinatewise.

Definition 3. Let $H_{i} \in(0,1), i=1,2$. A random Gaussian field such that

(1) $B_{0}^{H_{1}, H_{2}}=0$, E $B_{t}^{H_{1}, H_{2}}=0$;

(2) $\mathrm{E} B_{s}^{H_{1}, H_{2}} B_{t}^{H_{1}, H_{2}}=\prod_{i=1,2}\left(s_{i}^{2 H_{i}}+t_{i}^{2 H_{i}}-\left|t_{i}-s_{i}\right|^{2 H_{i}}\right)$, where $s=\left(s_{1}, s_{2}\right)$ and $t=\left(t_{1}, t_{2}\right)$

(3) the trajectories of the field $B^{H_{1}, H_{2}}$ are continuous with probability 1 is called a fractional Brownian field $\left\{B_{t}^{H_{1}, H_{2}}, t \in \mathbb{R}_{+}^{2}\right\}$ with Hurst indices $H_{1}$ and $H_{2}$.

In what follows we denote the Hurst index by $H=\left(H_{1}, H_{2}\right)$ and consider the case of $H_{i} \in\left(\frac{1}{2}, 1\right), i=1,2$. Note that a fractional Brownian field with $H_{1}=H_{2}=\frac{1}{2}$ is the Chentsov-Wiener field. 
Remark 8. According to a result of the paper [5], $B^{H} \in \mathcal{H}^{H_{1}-\varepsilon_{1}, H_{2}-\varepsilon_{2}}$ almost surely with respect to the measure $\mathrm{P}$ for all $0<\varepsilon_{i}<H_{i}, i=1,2$, where $B^{H}$ is a fractional Brownian field. Thus $B^{H} \in W_{2}^{1-\beta_{1}, 1-\beta_{2}}$ for all $1-\beta_{i}<H_{i}, i=1,2$, and $\Lambda_{1-\beta_{1}, 1-\beta_{2}}\left(B^{H}\right)<\infty$ almost surely with respect to the measure $P$.

Remark 9. The bounds obtained in Theorems 1 and 2 remain true in the case of

$$
f(s)=g(s)=B_{s}^{H}, \quad s \geq 0,
$$

and a fractional Brownian field has the Hurst indices $H_{i} \in\left(\frac{1}{2}, 1\right), i=1,2$.

Let $(\Omega, \mathcal{F}, \mathrm{P})$ be a complete probability space where a fractional Brownian field

$$
B_{t}^{H}:[0, T] \times \Omega \rightarrow \mathbb{R}
$$

is defined.

Consider a stochastic differential equation of the form

$$
X_{t}=X_{0}+\int_{[0, t]} b\left(s, X_{s}\right) d s+\int_{[0, t]} \sigma\left(s, X_{s}\right) d B_{s}^{H}=X_{0}+F_{t}^{(b)}(X)+G_{t}^{(\sigma)}(X),
$$

where $t \in[0, T] \subset \mathbb{R}_{+}^{2}$, a point $T$ is fixed, $B_{t}^{H}$ is a fractional Brownian field with Hurst indices $H_{i} \in\left(\frac{1}{2}, 1\right), i=1,2$, and $\sigma, b:[0, T] \times \mathbb{R} \rightarrow \mathbb{R}$ are measurable functions such that

$$
\left\{\begin{array}{l}
\text { 1) } b(s, x) \text { is continuous with respect to } s \text { and for all } N>0 \text { there exists } \\
C_{N}>0 \text { such that }|b(t, x)-b(t, y)| \leq C_{N}|x-y| \text { for all }|x|,|y| \leq N \\
\text { and all } t \in[0, T] ; \\
\text { 2) there exists } C>0 \text { such that }|b(t, x)| \leq C \text { and }|\sigma(t, x)| \leq C \text { for all } \\
t \in[0, T] \text { and } x \in \mathbb{R} ; \\
\text { 3) } \sigma \in C^{3}[0, T] \times C^{5}(\mathbb{R}) \text {; } \\
\text { 4) there exists } C>0 \text { such that }|D \sigma(t, x)| \leq C \text {, where } D \text { denotes any } \\
\text { operation of differentiation which is allowed according to condition 3). }
\end{array}\right.
$$

The random field

$$
X_{t}=X_{t}(\omega):[0, T] \times \Omega \rightarrow \mathbb{R}
$$

is called a solution of equation (26) if it turns equality (26) into an identity for almost all $\omega \in \Omega$ and if the integral $G_{t}^{(\sigma)}(X)$, treated in the sense of the generalized two-parameter Lebesgue-Stieltjes integral, is well defined for almost all $\omega \in \Omega$.

The proof of the main result of this section uses Lemma 7.2 of $[2$.

Lemma 1 ([2]). Let $(X, \rho)$ be a complete metric space and $\rho_{0}, \rho_{1}$, and $\rho_{2}$ be some metrics on $X$ that are equivalent to $\rho$. Assume that the transformation $\mathfrak{L}: X \rightarrow X$ is such that

(1) there exist $r_{0}>0$ and $x_{0} \in X$ for which

$$
\mathfrak{L}\left(B_{0}\right) \subset B_{0},
$$

where $B_{0}=\left\{x \in X: \rho_{0}\left(x_{0}, x\right) \leq r_{0}\right\}$;

(2) there exist a lower semi-continuous function $\varphi:(X, \rho) \rightarrow[0,+\infty]$ and a constant $C_{0} \geq 0$ such that

(a) $\varphi(x) \leq C_{0}$ for all $x \in \mathfrak{L}\left(B_{0}\right)$,

(b) $\rho_{1}(\mathfrak{L}(x), \mathfrak{L}(y)) \leq(\varphi(x)+\varphi(y)) \rho_{1}(x, y)$ for all $x, y \in \mathfrak{L}\left(B_{0}\right)$;

(3) there exists $a \in(0,1)$ such that

$$
\rho_{2}(\mathfrak{L}(x), \mathfrak{L}(y)) \leq a \rho_{2}(x, y) \quad \text { for all } x, y \in \mathfrak{L}\left(B_{0}\right) .
$$


Then there exists $x^{*} \in \mathfrak{L}\left(B_{0}\right) \subset X$ such that

$$
x^{*}=\mathfrak{L}\left(x^{*}\right) .
$$

Theorem 7. Let all assumptions 1)-4) in (27) hold for the coefficients of equation (26). Then equation (26) possesses a unique in the class $W_{1}^{\alpha_{1}, \alpha_{2}}$ solution $X_{t}$ on the rectangle $[0, T]$. Moreover

$$
X \in \mathcal{H}^{1-\alpha_{1}, 1-\alpha_{2}}
$$

for almost all $\omega \in \Omega$ and all $1-H_{i}<\alpha_{i}<\frac{1}{2}, i=1,2$.

Proof. Fix some $\omega \in \Omega$. In what follows we omit the argument $\omega$. Assume that

$$
X \in W_{1}^{\alpha_{1}, \alpha_{2}}
$$

is a solution of equation (26) for a given $\omega$ and for some $\alpha_{i} \in\left(1-H_{i}, \frac{1}{2}\right)$. By Theorems 2 and 5. $G^{(\sigma)}(X) \in \mathcal{H}^{1-\alpha_{1}, 1-\alpha_{2}}$ and $F^{(b)}(X) \in C^{1}$, whence

$$
X \in \mathcal{H}^{1-\alpha_{1}, 1-\alpha_{2}} .
$$

First we prove the uniqueness of a solution. Let $N>0$ be a number such that

$$
\|X\|_{1, \alpha_{1}, \alpha_{2}} \leq N, \quad\|\widetilde{X}\|_{1, \alpha_{1}, \alpha_{2}} \leq N .
$$

Then $\|X\| \vee\|\widetilde{X}\| \leq N$, where $\|X\|=\sup _{t \in[0, T]}\left|X_{t}\right|$ and

$$
\|X\|_{\alpha_{1}, \alpha_{2}, \lambda_{1} / 2, \lambda_{2} / 2}^{2} \leq C(1+\|N\|)\|X\|_{\alpha_{1}, \alpha_{2}, \lambda_{1}, \lambda_{2}} .
$$

Write

$$
X_{t}=X_{0}+F_{t}^{(b)}(X)+G_{t}^{(\sigma)}(X), \quad \widetilde{X}_{t}=X_{0}+F_{t}^{(b)}(\tilde{X})+G_{t}^{(\sigma)}(\tilde{X}) .
$$

If conditions 1), 3), and 4) of (27) hold for $\lambda_{i}>1, i=1,2$, then there exists a constant $C_{N, H, \alpha_{1}, \alpha_{2}, T}$ such that

$$
\begin{aligned}
\| X- & \tilde{X} \|_{\alpha_{1}, \alpha_{2}, \lambda_{1}, \lambda_{2}} \\
& \leq\left\|F^{(b)}(X)-F^{(b)}(\widetilde{X})\right\|_{\alpha_{1}, \alpha_{2}, \lambda_{1}, \lambda_{2}}+\left\|G^{(\sigma)}(X)-G^{(\sigma)}(\widetilde{X})\right\|_{\alpha_{1}, \alpha_{2}, \lambda_{1}, \lambda_{2}} \\
& \leq \frac{C_{N, H, \alpha_{1}, \alpha_{2}, T}}{\lambda_{1}^{1-2 \alpha_{1}} \lambda_{2}^{1-2 \alpha_{2}}}\left(\|X-\widetilde{X}\|_{\alpha_{1}, \alpha_{2}, \lambda_{1}, \lambda_{2}}\right)
\end{aligned}
$$

by Theorems 3 and 6 .

Choose $\lambda_{i}$ so large that

$$
\frac{C_{N, H, \alpha_{1}, \alpha_{2}, T}}{\lambda_{1}^{1-2 \alpha_{1}} \lambda_{2}^{1-2 \alpha_{2}}}<\frac{1}{2}
$$

Then we get $\frac{1}{2}\|X-\tilde{X}\|_{\alpha_{1}, \alpha_{2}, \lambda_{1}, \lambda_{2}} \leq 0$, whence $X_{t}=\widetilde{X}_{t}$ (we identify functions that are equal almost everywhere with respect to Lebesgue measure).

Now we prove the existence of a solution. Recall that $\omega \in \Omega$ is fixed. We rewrite equation (26) as follows:

$$
\widetilde{X}_{t}=\int_{[0, t]} \widetilde{b}\left(s, \widetilde{X}_{s}\right) d s+\int_{[0, t]} \tilde{\sigma}\left(s, \widetilde{X}_{s}\right) d B_{s}^{H},
$$

where $\widetilde{X}_{t}:=X_{t}-X_{0}, \widetilde{b}(s, x)=b\left(s, x+X_{0}\right)$, and $\widetilde{\sigma}(s, x)=\sigma\left(s, x+X_{0}\right)$.

We apply the fixed point theorem in the space $W_{1}^{\alpha_{1}, \alpha_{2}}$ but for another norm. Consider the operator

such that

$$
A: W_{1}^{\alpha_{1}, \alpha_{2}} \rightarrow \mathcal{H}^{1-\alpha_{1}, 1-\alpha_{2}} \subset W_{1}^{\alpha_{1}, \alpha_{2}}
$$

$$
\begin{gathered}
y(t)=(A x)(t)=\widetilde{F}_{t}^{(b)}(x)+\widetilde{G}_{t}^{(\sigma)}(x), \quad t \in[0, T], \\
\widetilde{F}_{t}^{(b)}(x)=F_{t}^{(\widetilde{b})}\left(x+X_{0}\right), \quad \widetilde{G}_{t}^{(\sigma)}(x)=G_{t}^{(\widetilde{\sigma})}\left(x+X_{0}\right) .
\end{gathered}
$$


Note that

$$
\|X\|_{\alpha_{1}, \alpha_{2}, \lambda_{1} / 2, \lambda_{2} / 2}^{2} \leq\|X\|_{\alpha_{1}, \alpha_{2}, \lambda_{1}, \lambda_{2}} \cdot\|X\|_{1, \alpha_{1}, \alpha_{2}} .
$$

By statement (2) of Theorem 2, Theorem [5] and Remarks 6 and 7 .

$$
\begin{aligned}
\|A x\|_{\alpha_{1}, \alpha_{2}, \lambda_{1}, \lambda_{2}} \leq & \left\|\widetilde{F}^{(\widetilde{b})}(x)\right\|_{\alpha_{1}, \alpha_{2}, \lambda_{1}, \lambda_{2}}+\left\|\widetilde{G}^{(\widetilde{\sigma})}(x)\right\|_{\alpha_{1}, \alpha_{2}, \lambda_{1}, \lambda_{2}} \\
\leq & \left(\frac{C_{0}}{\lambda_{1}^{1-\alpha_{1}} \lambda_{2}^{1-\alpha_{2}}}+\frac{C_{0} \Lambda_{\alpha_{1}, \alpha_{2}}\left(B^{H}\right)}{\lambda_{1}^{1-2 \alpha_{1}} \lambda_{2}^{1-2 \alpha_{2}}}(1+\|x\|)^{2}\left(1+\|x\|_{1, \alpha_{1}, \alpha_{2}}\right)\right) \\
& \times\left(1+\|x\|_{\alpha_{1}, \alpha_{2}, \lambda_{1}, \lambda_{2}}\right),
\end{aligned}
$$

since conditions 1)-4) of (27) hold where $C_{0}$ is a constant. We recall that $\alpha_{1}, \alpha_{2}$, and $T$ are fixed.

Note that $\|x\| \leq\|x\|_{1, \alpha_{1}, \alpha_{2}} \leq e^{\lambda_{1} T_{1}+\lambda_{2} T_{2}}\|x\|_{\alpha_{1}, \alpha_{2}, \lambda_{1}, \lambda_{2}}$. Thus

$$
\|A x\|_{\alpha_{1}, \alpha_{2}, \lambda_{1}, \lambda_{2}} \leq \frac{D_{0}}{\lambda_{1}^{1-2 \alpha_{1}} \lambda_{2}^{1-2 \alpha_{2}}}\left(1+\|x\|_{\alpha_{1}, \alpha_{2}, \lambda_{1}, \lambda_{2}} e^{\lambda_{1} T_{1}+\lambda_{2} T_{2}}\right)^{4}
$$

for $\lambda_{i}>1$. Let $\|x\|_{\alpha_{1}, \alpha_{2}, \lambda_{1}, \lambda_{2}} \leq 1$, where $\lambda_{1}=\lambda_{2}=\lambda$ is such that

$$
\frac{D_{0}}{\lambda^{2-2 \alpha_{1}-2 \alpha_{2}}}(1+2 e)^{4} \leq 1
$$

and $T_{i}^{0}=T_{0}:=1 /(2 \lambda), i=1,2$. Then $\|A x\|_{\alpha_{1}, \alpha_{2}, \lambda, \lambda} \leq 1$, that is,

$$
A\left(B_{0}\right) \subset B_{0},
$$

where $B_{0}=\left\{x \in W_{1}^{\alpha_{1}, \alpha_{2}}:\|x\|_{\alpha_{1}, \alpha_{2}, \lambda, \lambda} \leq 1\right\}$.

Considering the operator $A$ on the set $B_{0}$ and for the metric $\rho_{0}$ generated by the norm $\|\cdot\|_{\alpha_{1}, \alpha_{2}, \lambda, \lambda}$, we see that assumption (1) of Lemma 1 holds.

Theorems 3 and 6 imply that

$$
\begin{aligned}
\| A x- & A y \|_{\alpha_{1}, \alpha_{2}, \lambda_{1}, \lambda_{2}} \\
\leq & \frac{C}{\lambda_{1}^{1-2 \alpha_{1}} \lambda_{2}^{1-2 \alpha_{2}}}\left(1+\|x\|_{1, \alpha_{1}, \alpha_{2}}+\|y\|_{1, \alpha_{1}, \alpha_{2}}\right)^{5}\|x-y\|_{\alpha_{1}, \alpha_{2}, \lambda_{1}, \lambda_{2}} \\
& +\frac{C}{\lambda_{1}^{1-\alpha_{1}} \lambda_{2}^{1-\alpha_{2}}}\|x-y\|_{\alpha_{1}, \alpha_{2}, \lambda_{1}, \lambda_{2}}
\end{aligned}
$$

for all $x, y \in B_{0} \supset A\left(B_{0}\right)$ and for all $\lambda_{i} \geq 1$.

Note that $\varphi(x):=\left(1+\|x\|_{1, \alpha_{1}, \alpha_{2}}\right)^{5}$ is a continuous function on the space $W_{1}^{\alpha_{1}, \alpha_{2}}$ and moreover there exists $C_{0}>0$ such that $\varphi(x) \leq C_{0}$ for all $x \in A\left(B_{0}\right)$. The bound (29) implies that

$$
\rho_{1}(A x, A y) \leq C(\varphi(x)+\varphi(y)) \rho_{1}(x, y) \quad \text { for all } x, y \in A\left(B_{0}\right),
$$

where $\rho_{1}$ is the metric generated by the norm $\|\cdot\|_{\alpha_{1}, \alpha_{2}, 1,1}$. Thus assumption (2) of Lemma 1 holds.

If $\lambda_{1}$ and $\lambda_{2}$ are such that

$$
\frac{C}{\lambda_{1}^{1-2 \alpha_{1}} \lambda_{2}^{1-2 \alpha_{2}}}(1+2 e)^{5}+\frac{C}{\lambda_{0}^{1-\alpha_{1}} \lambda_{0}^{1-\alpha_{2}}} \leq \frac{1}{2},
$$

then $\|A x-A y\|_{\alpha_{1}, \alpha_{2}, \lambda_{0}, \lambda_{0}} \leq \frac{1}{2}\|x-y\|_{\alpha_{1}, \alpha_{2}, \lambda_{0}, \lambda_{0}}$. For $\lambda_{0}>\lambda$, we choose $T^{0}=1 /\left(2 \lambda_{0}\right)$. Then assumption (3) of Lemma 1 holds. This implies that the operator $A$ has a fixed point in the square $\left[0, T^{0}\right]^{2}$. Thus a solution exists in this square.

Now we consider equation (26) in the square $\left[T^{0}, 2 T^{0}\right] \times\left[0, T^{0}\right]$. We rewrite the equation in the following form:

$$
\widetilde{X}_{t}=\int_{[T, 2 T] \times[0, T]} \widetilde{b}\left(s, \widetilde{X}_{s}\right) d s+\int_{[T, 2 T] \times[0, T]} \widetilde{\sigma}\left(s, \widetilde{X}_{s}\right) d B_{s}^{H},
$$


where $\tilde{X}_{t}=X_{t}-X_{T^{0}, t_{2}}-X_{t_{1}, 0}+X_{T^{0}, 0}=X_{t}-X_{T^{0}, t_{2}}, \widetilde{b}(s, x)=b\left(s, x-X_{T^{0}, t_{2}}\right)$, and $\tilde{\sigma}(s, x)=\sigma\left(s, x-X_{T^{0}, t_{2}}\right)$.

One can apply the same reasoning to equation (30) as in the case of equation (28). As a result we prove the existence of a solution on $\left[T^{0}, 2 T^{0}\right] \times\left[0, T^{0}\right]$. Continuing the reasoning in this way we prove the existence of a solution on the whole interval $[0, T]$.

Remark 10. The existence and uniqueness of a solution of a stochastic differential equation with respect to a fractional Brownian field is proved in 2 in the one-parameter case.

\section{BIBLIOGRAPHY}

1. S. A. Il'chenko and Yu. S. Mishura, Generalized two-parameter Lebesgue-Stieltjes integrals and their applications to fractional Brownian fields, Ukrain. Mat. Zh. 56 (2004), no. 4, 435-450; English transl. in Ukrainian Math. J. 56 (2004), no. 4, 527-546. MR2105898 (2005i:60068)

2. D. Nualart and A. Răşcanu, Differential equations driven by fractional Brownian motion, Collect. Math. 53 (2002), no. 1, 55-81. MR1893308 (2003f:60105)

3. Yu. S. Mishura and S. A. Il'chenko, Some estimates for two-parameter generalized stochastic Lebesgue-Stieltjes integrals, Theory Stochastic Processes 9(25) (2003), no. 3-4, 87-100. MR 2306063

4. F. Russo and P. Vallois, The generalized covariation process and Itô formula, Stochastic Process. Appl. 59 (1995), 81-104. MR1350257 (96f:60089)

5. A. Kamont, On the fractional anisotropic Wiener field, Probab. Math. Statist. 16 (1996), no. 1, 85-98. MR1407935 (98a:60064)

Department of Probability Theory and Mathematical Statistics, Faculty for Mechanics and Mathematics, National Taras Shevchenko University, Academician Glushkov Avenue, 6, KYIV 03127, Ukraine

E-mail address: myus@univ.kiev.ua

Department of Probability Theory and Mathematical Statistics, Faculty for Mechanics and Mathematics, National Taras Shevchenko University, Academician Glushkov Avenue, 6, KYIV 03127, UkRAINE

E-mail address: ilchenko_sv@univ.kiev.ua

Received 17/OCT/2005

Translated by O. I. KLESOV 